\title{
Gain-Scheduled Linear Fractional Control for Active Flutter Suppression
}

\author{
Jeffrey M. Barker* and Gary J. Balas ${ }^{\dagger}$ \\ University of Minnesota, Minneapolis, Minnesota 55455 \\ and \\ Paul A. Blue \\ U.S. Air Force Research Laboratory, Wright-Patterson Air Force Base, Ohio 45433
}

\begin{abstract}
A gain-scheduled controller for active flutter suppression of the NASA Langley Research Center's Benchmark Active Controls Technology wing section is presented. The wing section changes significantly as a function of Mach and dynamic pressure and is modeled as a linear system whose parameters depend in a linear fractional manner on Mach and dynamic pressure. The resulting gain-scheduled controller also depends in a linear fractional manner on Mach and dynamic pressure. Stability of the closed-loop system over a wide range of Mach and dynamic pressure is demonstrated. Closed-loop stability is demonstrated via time simulations in which both Mach and dynamic pressure are allowed to vary in the presence of input disturbances. The linear fractional gain-scheduled controller and an optimized linear controller (designed for comparison) both achieve closed-loop stability, but the gain-scheduled controller outperforms the linear controller throughout the operating region.
\end{abstract}

\section{Nomenclature}

\begin{tabular}{|c|c|}
\hline$A, B, C, D$ & $=$ state-space representation of a system \\
\hline$D$ & $=$ stable, rational polynomial for $\mu$ design \\
\hline$e$ & $=$ generic element of a state-space matrix \\
\hline$F_{u}, F_{l}$ & $=$ upper and lower linear fractional transformations \\
\hline$I^{u}$ & $=$ identity matrix \\
\hline$K$ & $=$ controller \\
\hline$M$ & $=$ Mach number \\
\hline$N$ & $=$ constant $2 \times 2$ block matrix \\
\hline $\boldsymbol{P}$ & $=$ parameter dependent plant \\
\hline $\bar{q}$ & $=$ dynamic pressure, $\mathrm{kPa}$ \\
\hline$u$ & $=$ control signal \\
\hline$y$ & $=$ measured plant variables \\
\hline$\Delta$ & $=$ uncertainty model \\
\hline$\delta_{M}$ & $=$ normalized variation in Mach \\
\hline$\delta_{\bar{q}}$ & $=$ normalized variation in dynamic pressure \\
\hline$\mu$ & $=$ structured singular value \\
\hline$\Sigma$ & $=$ summation \\
\hline
\end{tabular}

Subscripts

$\begin{array}{ll}\text { act } & =\text { actuator } \\ d & =\text { disturbance } \\ \text { in } & =\text { input (to plant) multiplicative } \\ \text { LFT } & =\text { linear fractional transformation } \\ n & =\text { noise } \\ p & =\text { performance }\end{array}$

\section{Introduction}

$\mathbf{F}$ LUTTER is a dynamic instability, characterized in detail in 1935 (Ref. 1) and written of as early as 1916 (Ref. 2), that can resultin catastrophic mechanical failure of an aircraft wing. Because of the severity of the potential problem, aircraft today typically operate at conditions well below the flutter boundary. However, as aircraft design moves toward lighter weight materials in efforts to

Received 8 June 1998; revision received 21 December 1998; accepted for publication 22 December 1998. Copyright (C) 1999 by the authors. Published by the American Institute of Aeronautics and Astronautics, Inc., with permission.

* Graduate Research Assistant, Department of Aerospace Engineering and Mechanics, 110 Union Street SE. Student Member AIAA.

${ }^{\dagger}$ Associate Professor, Department of Aerospace Engineering and Mechanics, 110 Union Street SE. Member AIAA.

$\stackrel{+}{\dagger}$ First Lieutenant, U.S. Air Force, Flight Control Division. improve fuel efficiency and aircraft agility, active flutter suppression will likely become increasingly important.

The problem of active flutter suppression thus has received much attention in the form of the active flexible wing (AFW) program ${ }^{3,4}$ and the Benchmark Active Controls Technology (BACT) wing, developed at NASA Langley Research Center specifically to better understand flutter and its suppression. ${ }^{5-11}$ The AFW has been used for testing various single-input/single-output and multi-input/ multi-output controllers. ${ }^{12}$ The BACT model has been used to develop adaptive neural control schemes ${ }^{13}$ and generalized predictive control. ${ }^{14}$ Other control approaches to active flutter suppression that have been investigated include optimal control using acoustics, ${ }^{15}$ multirate control, ${ }^{16}$ nonlinear control, ${ }^{17}$ and $H_{2} / H_{\infty}$ control. ${ }^{18,19}$

This paper focuses on the design of an active flutter suppression gain-scheduled controller using linear fractional transformations (LFT). Gain-scheduled LFT control is a natural extension of $H_{\infty}$ control for systems that vary smoothly as a function of measurable parameters. Gain-scheduledLFT controller synthesis has been successfully applied to a wide variety of problems with parameter dependent plants, including lateral-directional control of the F-14 aircraft during powered approach. ${ }^{20}$ This control methodology is applied here to the BACT model. Scheduling the controller as a function of Mach and dynamic pressure allows the smooth handling of transitions in the dynamic model throughout the operating envelope. The gain-scheduled controller presented has a designed operating range of Mach from 0.5-0.82 and a dynamic pressure of $6.5-10.77 \mathrm{kPa}$. The range of operating conditions includes most of the unstable operating region and demonstrates how a single gain-scheduled LFT controller can stabilize the BACT model for a wide range of Mach and dynamic pressure. In addition to the gainscheduled controller, a single linear controller is designed using a $D-K$ iteration technique, which attempts to be robust to the changing flutter dynamics. Comparison of the two controllers demonstrates the improved performanceobtainable through use of gain-scheduled control techniques.

The paper is presented in the following six sections. Section II describes the BACT facility, the wing section test bed, and the linear, time-invariant (LTI) models derived at specific Mach numbers and dynamic pressures. In Sec. III an LFT model of the BACT wing section, which is a function of $M$ and $\bar{q}$, is derived from the LTI models. This LFT model is used for control design. Section IV presents the control theory associated with gain-scheduledLFT control. The gain-scheduled control problem requires the solution of linear matrix inequalities that can be solved efficiently ${ }^{21,22}$ using convex optimization techniques. Control design and synthesis of 
Table 1 Flight conditions of LTI models

\begin{tabular}{lllllllll}
\hline \hline Mach & \multicolumn{7}{c}{ Dynamic pressure, kPa } \\
\hline 0.50 & 3.59 & 4.79 & $5.84^{\mathrm{a}}$ & $6.32^{\mathrm{a}}$ & $7.18^{\mathrm{a}}$ & $8.38^{\mathrm{a}}$ & $9.58^{\mathrm{a}}$ & $10.77^{\mathrm{a}}$ \\
0.70 & 3.59 & 4.79 & 5.99 & $6.51^{\mathrm{a}}$ & $6.99^{\mathrm{a}}$ & $8.38^{\mathrm{a}}$ & $9.58^{\mathrm{a}}$ & $10.77^{\mathrm{a}}$ \\
0.78 & 3.59 & 4.79 & 5.99 & $6.75^{\mathrm{a}}$ & $7.22^{\mathrm{a}}$ & $8.38^{\mathrm{a}}$ & $9.58^{\mathrm{a}}$ & $10.77^{\mathrm{a}}$ \\
0.82 & 3.59 & 4.79 & 5.99 & $6.84^{\mathrm{a}}$ & $7.33^{\mathrm{a}}$ & $8.38^{\mathrm{a}}$ & $9.58^{\mathrm{a}}$ & $10.77^{\mathrm{a}}$ \\
\hline \hline
\end{tabular}

${ }^{\mathrm{a} O p e n-l o o p ~ u n s t a b l e . ~}$

the gain-scheduled LFT controller and a linear $\mu$ controller for the BACT model are presented in Sec. V. The controllers designed in Sec. V are analyzed and simulated in Sec. VI. The stability and performance of both the gain-scheduleddesign and the $\mu$ controller are evaluated over varying $M$ and $\bar{q}$. These results of the simulations are then compared and contrasted. The final section summarizes the results and presents conclusions.

\section{BACT Model}

The BACT model is an element of NASA Langley Research Center's Benchmark Models Program (BMP). ${ }^{5}$ The BMP consists of several models used in the investigation of aeroelastic effects and to acquire experimental data for the validation of the computational fluid dynamics (CFD) code. The BACT model has been used to obtain experimental data over a wide range of operating conditions, which researchers use to develop active flutter suppression design tools and to calibrate unsteady CFD code.

The BACT model is a rigid, rectangularwing with an NACA 0012 airfoil. $^{8}$ The airfoil has three control surfaces driven by hydraulic actuators: a trailing-edge flap with a \pm 15 -deg operating range and upper and lower surface spoilers, each with a range from 0 to $45 \mathrm{deg}$. The primary sensors used for control are four linear accelerometers, placed at the four corners of the wing section. The wing model is mounted on a flexible device called the Pitch and Plunge Apparatus (PAPA). PAPA is designed to allow rotation (pitch) and vertical translation (plunge) ${ }^{7,8}$

Using Interaction of Structures, Aerodynamics, and Controls (ISAC), ${ }^{23,24}$ a computer program for calculating the interactive effects of flexible structures, unsteady aerodynamics, and active controls, NASA researchers developed LTI models for the BACT windtunnel model at a wide range of operating conditions. The program uses a doublet-lattice method to calculate the three-dimensional aerodynamic forces on the wing for a specified operating condition. LTI models have been generated to represent the wing at various wind-tunnel operating conditions. The LTI models made available (Table 1) are at four different Mach numbers and range in dynamic pressure from 3.59 to $10.77 \mathrm{kPa}$. These LTI models have only one control input (the trailing-edge flap) and leading- and trailing-edge acceleration measurements. The LTI models have 14 states: four states correspond to the pitch and plunge dynamics, six states characterize unsteady aerodynamics, two states characterize actuator dynamics, and two states are a second-order Dryden turbulence model.

Examination of Bode plots of the LTI models reveals that they are primarily functions of the pitch, plunge, and actuator states, with the unsteady aerodynamics playing a small role and the gust model resulting in a small disturbance. Figure 1 shows representative magnitude plots for the full (14th) order and reduced (6th) order LTI systems. Clearly, the salient features of the flutter problem for the BACT wing are captured in the reduced-order systems. Essentially, the BACT wing appears to be well represented by a model similar to a wing model with quasisteady aerodynamics. This is not surprising because the wing was designed so that the aerodynamic instabilities would be relatively benign, which made building in safety mechanisms easier. ${ }^{14}$

\section{LFT Model Synthesis}

The implementation of gain-scheduled LFT control requires the construction of an LFT plant from the BACT LTI model data. The 32 LTI models of Table 1 are the basis for the LFT model of the system used for control design. (The operating range of the LFT model used for control design includes the 19 models at dynamic
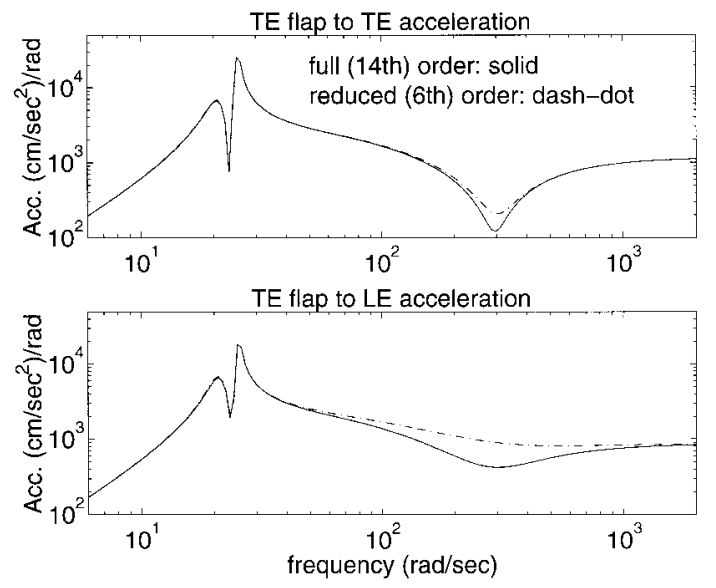

Fig. 1 Full-and reduced-order transfer function models from trailingedge actuator to trailing- and leading-edge accelerometers at $\bar{q}=$ $8.38 \mathrm{kPa}$ and $\mathrm{Mach}=0.7$.

pressures of greater than $6.5 \mathrm{kPa}$.) The model, previously described in detail, ${ }^{25}$ is presented here in a much abbreviated format.

First, a model depending on Mach and dynamic pressure was developed in which each element of the state matrices $(\boldsymbol{A}, \boldsymbol{B}, \boldsymbol{C}, \boldsymbol{D})$ is a function of $M$ and $\bar{q}$. The two state equations corresponding to the actuator model are constant across the 32 LTI models, and the pitch and plunge state equations are just integrations of the pitch-rate and plunge-ratestates. Each element $(e)$ of the remaining state-space equations could be accurately modeled over the range of operating conditions in Table 1, as

$$
e(\bar{q}, M)=e_{o}+e_{\bar{q}} \cdot \bar{q}+e_{M} \cdot M+e_{\bar{q} M} \cdot \bar{q} \cdot M
$$

This relationship allows the 32 LTI models to be written as one parameter-varying model of the form

$$
\begin{aligned}
& \dot{x}=\boldsymbol{A}(\bar{q}, M) \cdot x+\boldsymbol{B}(\bar{q}, M) \cdot u \\
& y=\boldsymbol{C}(\bar{q}, M) \cdot x+\boldsymbol{D}(\bar{q}, M) \cdot u
\end{aligned}
$$

where $\boldsymbol{A}(\bar{q}, M), \boldsymbol{B}(\bar{q}, M)$, etc., take the form of Eq. (1). Further study of the models reveals that $\boldsymbol{C}(\bar{q}, M)$ and $\boldsymbol{D}(\bar{q}, M)$ can be constructed from $\boldsymbol{A}(\bar{q}, M)$ and $\boldsymbol{B}(\bar{q}, M)$ because the outputs are accelerations (linear combinations of the state rates).

To ascertain the accuracy of the parameter-varyingrepresentation [Eqs. (2) and (3)], its poles were compared with the poles of the individual LTI models at the 32 flight conditions and were found to be very similar. ${ }^{25}$ Indeed, the parameter-varyingmodel was constructed to match the LTI models exactly at Mach 0.5 and 0.82 and is a good representation at intermediate Mach. Thus, the parameter-varying model accurately describes the LTI flutter models. This parametervarying model is used as the basis for the reduced-orderLFT model of the system used in control design.

Using the information from Fig. 1, one can see that a sixth-order model captures the primary effects of wing flutter for the BACT wing. Using a reduced-ordermodel of the plant so that the resulting controllerwill also be of low order is desirable; thus, the six unsteady aerodynamic states and the second-order Dryden wind-gust model are truncated from the model because they have little effect on the dynamics of the system. For modeling purposes the wind gust is replaced with an input disturbance injected on the actuator position. Although this does not accurately replace the effects of the gust model, the added disturbance path provides for added robustness. Thus, the single-input, two-output design model contains six states: the four states associated with the pitch and plunge modes and the two-state actuator model.

Before introducing the LFT model, some background on LFTs is appropriate. The upper and lower linear fractional transformations of a block-partitioned matrix

$$
N=\left[\begin{array}{ll}
N_{11} & N_{12} \\
N_{21} & N_{22}
\end{array}\right]
$$



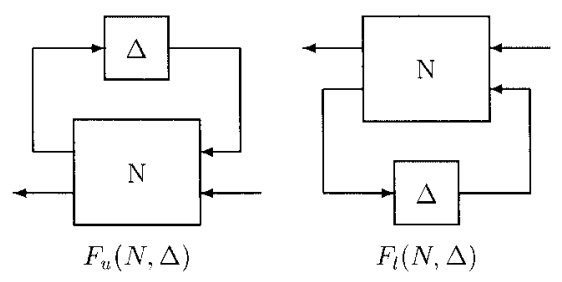

Fig. 2 Upper and lower linear fractional transformations.

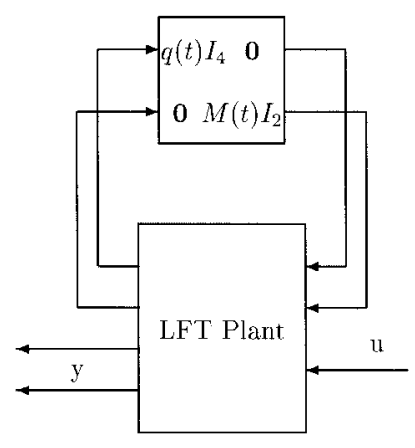

Fig. 3 LFT plant as a function of $\bar{q}$ and $M$.

with a matrix $\Delta$ are defined as

$$
\begin{aligned}
& F_{u}(N, \Delta)=N_{22}+N_{21} \Delta\left(I-N_{11} \Delta\right)^{-1} N_{12} \\
& F_{l}(N, \Delta)=N_{11}+N_{12} \Delta\left(I-N_{22} \Delta\right)^{-1} N_{21}
\end{aligned}
$$

shown in a block-diagram representation in Fig. 2.

Equation (1) can be used to formulate the parameter-varyingproblem in LFT form ${ }^{25}$ with four copies of the parameter $\bar{q}$ and two copies of $M$. Figure 3 shows the resulting LFT plant $\boldsymbol{P}$ as a function of $\bar{q}$ and $M$, which vary from $3.59-10.77 \mathrm{kPa}$ and from Mach $0.5-0.82$, respectively. Thus the parameterizationincludes both the open-loop stable and unstable models. (Recall that although $H_{\infty}$ control theory specifies stable perturbations, a stable $\Delta$ can either stabilize or destabilize given plant.) For control design purposes scaling $\bar{q}$ and $M$ so that they vary between -1 and 1 is necessary. To do this, a constant LFT $N$ is employed:

$$
F_{u}\left\{N,\left[\begin{array}{cc}
\delta_{\bar{q}}(t) & 0 \\
0 & \delta_{M}(t)
\end{array}\right]\right\}=\left[\begin{array}{cc}
\bar{q}(t) I_{4} & 0 \\
0 & M(t) I_{2}
\end{array}\right]
$$

The same process could be applied to the 14 state LTI models with additional copies of parameters $\bar{q}$ and $M$. The choice to use a six-state LFT model is thus one of engineering judgement, and the resulting controllers will of course be validated against the full-order LTI models.

The gain-scheduled LFT control design methodology is a direct extension of $\mu$-synthesis theory. Thus $\mu$ synthesis is a natural choice for the linear control design technique. Because the control problem in the $\mu$ framework is posed as maximizing robust performance, this is equivalent to minimizing the structured singular value $\mu$. Thus finding a controller $K$ to minimize $\mu$ is a reasonable objective. An approximationto the $\mu$-synthesis problem is given by the $D$ - $K$ iteration, which is a two-step iterative solution method. First a controller $K$ is found through standard $H_{\infty}$ methods. Next, the scaling matrix $D(s)$ that minimizes $\left\|D(s) F_{l}(P, K)(s) D^{-1}(s)\right\|_{\infty}$ is determined. These steps are iterated until an acceptable controller is obtained. Further details of the structured singular value theory and $D-K$ iteration may be found elsewhere. ${ }^{26-28}$

\section{Gain-Scheduled LFT Control}

There are two main performance objectives for any flutter suppression system. The first is to extend the flutter boundary, i.e., to use feedback control to stabilize the wing over a larger region of operating conditions. Second, flutter control is used to suppress vibrations in the operating region where the wing is open-loop stable. Extending the flutter boundary implies that the boundary is a function of measurable parameters (e.g., $M$ and $\bar{q}$ ). The variation of the plant as a function of one or more parameters is an essential feature of the problem. By allowing the controller to depend explicitly on these

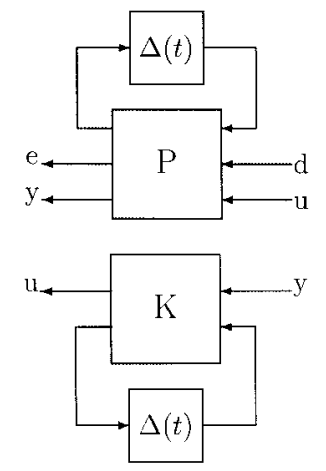

Fig. 4a Parameter-dependent plant.

Fig. 4b Parameter-dependent controller.

parameters, attaining improved closed-loopperformance and stability should be possible. Because the essential dynamics of the flutter problem are well described by the LFT plant, using gain-scheduled LFT controller synthesis techniques for active flutter suppression of the BACT model is appropriate.

The central idea of gain-scheduled LFT control is that a plant often can be represented as a linear fractional transformation of a nominal plant and physical parameters that vary within a known range. If we can measure these physical parameters in real time, then the controller can use this knowledge to schedule as a function of these parameters.

Consider a parameter-dependent plant modeled as an LFT of a time-varying block diagonal matrix $\Delta(t)$ and a three-input, threeoutput LTI plant $\boldsymbol{P}$ (Fig. 4a). The parameter dependence of the system is because of the time-varying $\Delta$ matrix. For the flutter problem the $\Delta(t)$ block is

$$
\Delta(t)=\left[\begin{array}{cc}
\delta_{\bar{q}}(t) I_{4} & 0 \\
0 & \delta_{M}(t) I_{2}
\end{array}\right]
$$

The assumption is made that $\Delta(t)$ takes values in a known set $\Delta$ and that $\Delta(t)$ can be measured in real time.

The parameter-dependentcontroller is restricted to having a structure similar to that of the plant (Fig. 4b). By interconnecting the parameter-dependent plant and controller the closed-loop system appears as a finite dimensional LTI system subjected to the timevarying perturbation $\Delta(t)$. The perturbation has a structure consisting of two parts: the physical parameters that affect the plant and the measured parameters that are used by the controller. The actual and measured parameters may be different, but for notational simplicity $\Delta(t)$ will be used to represent both.

The control objective is to design the controller $K_{\mathrm{LFT}}$ such that for all allowable perturbations $\Delta(t) \in \Delta$ the parameter dependent closed-loop system is internally exponentially stable with small induced $\mathcal{L}_{2}$ norm from disturbances to errors (including measurement noise and bounds on control authority). The small-gain theorem can be employed to bound (conservatively) the stability of the system and the induced $\mathcal{L}_{2}$ norm of the disturbance to error channels of the parameter-dependent closed-loop system. Scaling matrices are restricted to be constant diagonal matrices so that they will commute with the repeated structure of the perturbation. The main result of the theory that allows the determination of such a controller is that existence of a controller satisfying the scaled small-gain bound can be expressed exactly as the feasibility of a finite dimensional affine matrix inequality ${ }^{1}$ (AMI). Because of the convexity of the AMI, this problem can be computed numerically. The details of gainscheduled LFT control theory are covered in detail by Packard. ${ }^{29}$

\section{Control Design and Synthesis}

The control design methodology is very similar for both the gainscheduled LFT controller and the $\mu$ controller, and so will be presented only once in Sec. V.A. Controller synthesis is discussed for each method in Sec. V.B.

\section{A. Control Design}

The reduced six-state LFT plant (4 aerodynamic states, 2 actuator states) is used to design both flutter suppression controllers. This reduced plant has seven inputs and eight outputs. The first six inputs 


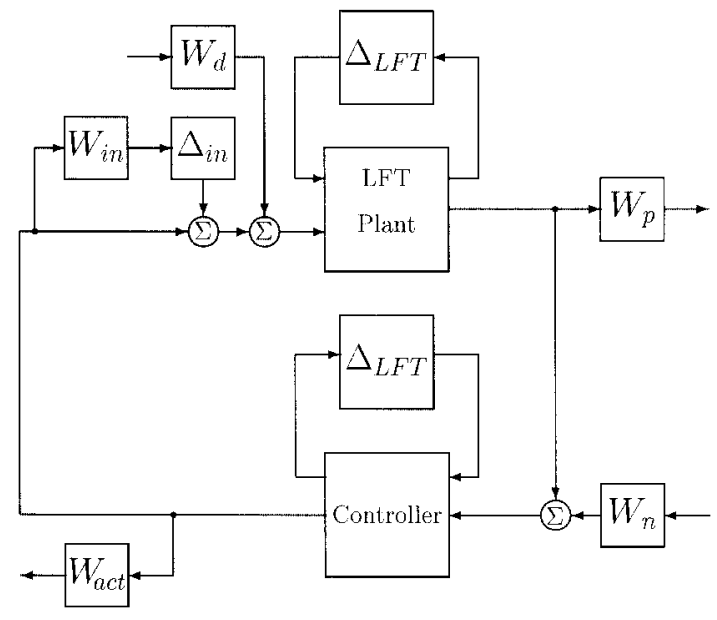

Fig. 5 LFT control design block diagram.

and outputs connect to the parameter block $\Delta(t)$ as discussed in Sec. IV. The other input is the control signal, and the two outputs, trailing-edge and leading-edge acceleration, are used for feedback.

The block diagram (Fig. 5) is used in the synthesis of both the LFT gain-scheduled controller and the $\mu$ controller. This diagram corresponds to the integration of performance objectives and robust stability objectivesinto a single control design framework. The stability objectives are to stabilize the wing throughout the operating region and to be robust to uncertainty in the modeling process and to errors in model reduction. These objectives are incorporated through both input multiplicative uncertainty and the LFT model (which enters as parametric uncertainty in the $\mu$ framework). Performance requirements are formulated through the choice of the weighting functions applied to the input and output signals of the open-loop system. The output leading- and trailing-edge accelerations are the primary performance signals. The flap command signal from the controller is also restricted. A disturbance signal on the input to the open-loop plant allows for unknown exogenous disturbances to the system, representing wind gusts. A noise signal is also added to the leadingand trailing-edge signals to corrupt the measurements.

Multiplicative uncertainty, represented in the block diagram by the multiplicativeuncertainty weight $W_{\text {in }}$ and the uncertainty set $\Delta_{\text {in }}$, is used to capture modeling error at high frequency, differences between the LFT model and the individual LTI models, and the uncertainty introduced by model reduction. For this system the multiplicative uncertainty weight used is $W_{\text {in }}=[0.1(s / 2)+1] /[(s / 200)+1]$, representing $10 \%$ uncertainty in the LFT model at low frequencies, $100 \%$ uncertainty at $20 \mathrm{rad} / \mathrm{s}$, increasing to $1000 \%$ at high frequencies. The level of uncertainty at high frequency ensures that the controllers will not be amplifying the system dynamics in this frequency range.

The LFT model, which is a function of $\bar{q}$ and $M$, is treated as parametric uncertainty in the $\mu$-synthesis control design. Thus the nominal plant for the linear control design is simply the plant that corresponds to $\delta_{\bar{q}}=0, \delta_{M}=0$ (correspondingto $7.18 \mathrm{kPa}$ and Mach 0.66 , an unstable open-loop plant), whereas in the LFT gain-scheduled design the variation in $\bar{q}$ and $M$ is part of the model.

The primary performance objective for active flutter suppression is to decrease the peak response of the wing at the flutter frequencies and the pitch and plunge modes. This objective is captured via a constant diagonal performance weighting $W_{p}$, which restricts the maximum magnitude of the transfer functions. The constants are first chosen to normalize the output channel (in $\mathrm{cm} / \mathrm{s}^{2} / \mathrm{rad}$ ) to have a peak value of approximately one (approximately, because the peak values of the transfer functions from flap command to leading- and trailing-edge acceleration vary as functions of $\bar{q}$ and M). These constants are then multiplied by two, asking that the peak magnitude be reduced to half of its initial value. Thus,

$$
W_{p}=2\left[\begin{array}{cc}
\frac{1}{27,500} & 0 \\
0 & \frac{1}{25,000}
\end{array}\right]
$$

is chosen. These constant weights, applied to the trailing- and leading-edge acceleration output channels, request a reduction of the maximum singular values from all inputs (disturbance on the actuator signal, uncertainty, and noise) to these outputs. Thus these weights correspond to asking for a decrease to $50 \%$ of the openloop peak response at the natural frequencies for the stable plants and to similar magnitudes at the flutter frequency for the open-loop unstable plants. Because the basic performance problem is one of vibration attenuation, the constant performance weight is all that is needed and is selected to suppress the peak singular values. Additionally, by choosing a constant performance weight, the order of the gain-scheduledLFT controller is kept low. (A first-order performance weight on each output channel would add two states to the controller, for example.)

The trailing-edge flap used as the actuator has limits of $\pm 15 \mathrm{deg}$ or $\pi / 12 \mathrm{rad}$. As in the performance weight, the actuator weight $W_{\text {act }}=12 / \pi$ is chosen to scale the largest allowable actuator command to \pm 1 . No rate limits are imposed on the actuator in this control design formulation although the high frequency gain of the multiplicative uncertainty effectively limits the bandwidth of the controller.

Sensor noise is added to the feedback signals to corrupt the measurements and to satisfy the $H_{\infty}$ control algorithm used for design. The weight $W_{n}=\operatorname{diag}[250,250]$ was chosen so that the maximum noise to signal ratio is about $10 \%$ in the frequency range $10-50 \mathrm{rad} / \mathrm{s}$.

Disturbances are introduced through a weighted disturbance input on the actuator command to the plant. The disturbance weight is chosen as $W_{d}=\pi / 36 \mathrm{rad}$, which represents maximum actuator positioning error on the order of $\frac{1}{3}$ the size of the maximum allowable actuator command. This constant disturbance weight was used for control design rather than using the Dryden gust model, which would add two states to the plant and introduce additional copies of the LFT parameters to the problem. The added disturbance model helps ensure the resulting controller is not overly aggressive in its attempt to damp out vibrations. The resulting controllers will be tested against the full-order system with the gust model included to validate the designs.

\section{B. Controller Synthesis}

The linear $\mu$ contoller $\left(K_{\mu}\right)$ was synthesized via the $D-K$ iterative control design technique using the MATLAB ${ }^{\mathrm{TM}} \mu$-Analysis and Synthesis Toolbox. ${ }^{26}$ The weighted open-loop system has seven states (the reduced six-state system plus one state for the multiplicative uncertainty weight). The structured uncertainty is introduced as a complex parameter variation. To keep the total order of the $\mu$ controller low, the four copies of the parameter $\bar{q}$ are allowed to vary independently (adding additional conservatism to the control design) whereas the two copies of $M$ vary together. Three iterations resulted in a 43rd-order controller, and further iterations decreased $\mu$ by less than $1 \%$. A balanced realization of the controller is obtained, and the 29 states with the smallest Hankel singular values are truncated from the system. The resulting 14-state controller differs from the full-order controller in $\mathcal{H}_{\infty}$ norm by less than $0.1 \%$. $\left(\mathcal{H}_{2}\right.$ norms cannot be compared because the high frequency gain of the reduced-ordercontrolleris small but constant, resultingin an infinite $\mathcal{H}_{2}$ norm.) The resulting reduced-order controller has $\mu<2.7$.

A gain-scheduled LFT controller $\left(K_{\mathrm{LFT}}\right)$ was synthesized using an algorithm that allows the parameters $\delta_{\bar{q}}$ and $\delta_{M}$ to be complex, which is conservative. $K_{\mathrm{LFT}}$ stabilizes the open-loopLFT plant over the full range of Mach (0.5-0.82) and a reduced range of dynamic pressures $(6.5-10.77 \mathrm{kPa})$. The gain-scheduledcontroller is synthesized using a formulation of the $H_{\infty}$ problem as a linear objective minimization. ${ }^{29}$ The resulting controllers have the same number of states as the weighted open-loop system. Thus the resulting controller is seventh order. A fourth-order truncated balanced realization of the controller results in a reduced-order controller differing from the original controller in $\mathcal{H}_{\infty}$ norm by less than $0.1 \%$ in the frequency range of interest.

Because of the structure of the problem, using the frequencyvarying $D$ scalings on the LFT parameters in the manner used in the $D-K$ iteration ${ }^{26}$ to determine the robust stability of the system is not possible. However, we are able to use a constant $D$ scaling calculated just below the flutter frequency at $20 \mathrm{rad} / \mathrm{s}$ and get an approximation 
of robust stability information. Performing the preceding scaling, then calculating frequency-dependent scalings for only the multiplicative uncertainty channel, a structured singular value of under 14 is obtained. The high value of $\mu$ is consistent with the $\mu$ value satisfied by the $\mu$-synthesis controller after the first iteration.

\section{Results}

The primary objectives were to improve the disturbance rejection characteristics of the wing and increase the range of operating conditions at which the wing is stable. In examining the success of the gain-scheduled controller at meeting these goals, several performance characteristics are considered. First, the stability of the closed-loopsystem is examined using the full-orderLTI single-point models and the point controllers obtained by specifying constant dynamic pressure and Mach. For example, the full-order (14 state) LTI model at Mach 0.7 and $10.77 \mathrm{kPa}$ was closed with $K_{\mathrm{LFT}}$ operating at the same Mach and $\bar{q}$. Second, the maximum singular value plots of the open- and closed-loop systems are compared. Finally, time simulations of the response of the wing to various inputs are used to examine the disturbance rejection characteristics, as well as to demonstrate reasonable actuator usage.

The gain-scheduled controller stabilizes the LFT plant over the range indicated in Sec. V.B. It remains to be shown that the controller also stabilizes all of the full-orderLTI plants within this range $(\bar{q}>6.5$ and $0.5<M<0.82)$. For $K_{\mathrm{LFT}}$, there are 19 LTI plants to be examined (Table 1). All 16 of the unstable LTI plants in this range are stable when closed with the LTI controllerobtained from the LFT controller $K_{\mathrm{LFT}}$ at the appropriate Mach and dynamic pressure. The three open-loop stable plants in the region remain stable. Additionally, although $K_{\mathrm{LFT}}$ was designed over only part of the operating range, it actually stabilizes and improves disturbance rejection over all 32 full-orderLTI models. For $K_{\mathrm{LFT}}$, this means that the point controller at a fixed Mach $M_{0}$ and $6.5 \mathrm{kPa}$ stabilizes the LTI model at $M_{0}$ and $3.59 \mathrm{kPa}$ and rejects disturbancesbetter than the open-loopplant at Mach 0.5 and $3.59 \mathrm{kPa}$. The gain-scheduled controller thus shows a greater than $50 \%$ increase in the flutter boundary as a function of dynamic pressure, which may indicate that a single gain-scheduled LFT controller operating over the entire range of LTI plants could be synthesized, perhaps by using real-valued (instead of complex) parameters in the LFT synthesis problem.

The linear $\mu$ controller $K_{\mu}$ also stabilizes the closed-loop system over all of the operating conditions. To accomplishthis, performance is traded off for stability, as will be shown next.

In evaluating the success of controllers in attenuating vibrations of flexible structures, examining the magnitude of the transfer functions or the maximum singular value plots of the system is typical. These generally give a good indication of how well the closed-loop systems will reject disturbances, especially in comparison to the open-loop plant.

Figure 6 shows the full-order LTI open-loop plant and the plant closed with $K_{\mathrm{LFT}}$ and $K_{\mu}$ at the two unstable extremes of the range of operating conditions (at high dynamic pressure). The two left-hand
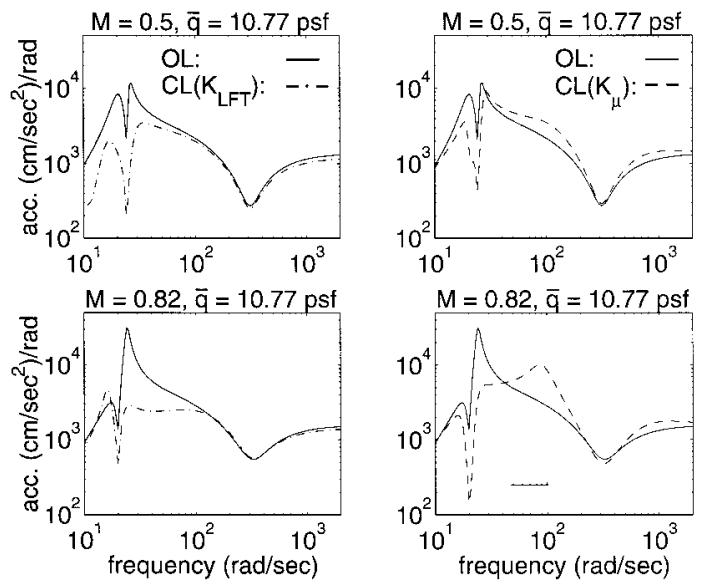

Fig. 6 Open- and closed-loop maximum singular value plots from trailing-edge flap to trailing- and leading-edge acceleration for both controllers. plots show maximum singular value plots of the open-loop plant and the plant closed with $K_{\mathrm{LFT}}$, whereas the right-hand plots show the open-loop plant at the same operating points closed with $K_{\mu}$. The upper plots are maximum singular value plots for the system at Mach 0.5 and $10.77 \mathrm{kPa}$ for each of the controllers, and the lower plots give the same at Mach 0.82 and $10.77 \mathrm{kPa}$. The first peak, at approximately $20 \mathrm{rad} / \mathrm{s}$, corresponds to the pitch mode in the stable operating range whereas the second, at approximately $26 \mathrm{rad} / \mathrm{s}$, corresponds to the plunge mode in the open-loopstable range. The mode that becomes unstable at the flutter boundary (at $26 \mathrm{rad} / \mathrm{s}$ ) exhibits characteristicsof both pitch and plunge, indicating that the pitch and plunge modes are coupled. This instability occurs at $6.08 \mathrm{kPa}$ for Mach 0.5 and at $7.09 \mathrm{kPa}$ for Mach 0.82 . The significant reduction in the peak singular values with $K_{\mathrm{LFT}}$ at both Mach 0.5 and 0.82 and $10.77 \mathrm{kPa}$ is representative of the reductions seen at other operating points, indicating that good vibration attenuation/disturbance rejection throughout the operating range is likely. Time simulations will show that these disturbance rejection conclusions are valid. $K_{\mu}$ also attenuates the peaks at both operating conditions, but by significantly less than $K_{\mathrm{LFT}}$; this suggests that while the $\mu$-synthesis controller stabilizes the plant throughout the operating range the disturbance attenuation will be significantly smaller at the extremes of the operating range than that achieved by $K_{\mathrm{LFT}}$.

Time simulations of the closed-loop systems are used to investigate both the response of the system to input disturbances and the stability of the closed-loop systems as $\bar{q}$ and Mach vary as functions of time. Figure 7 demonstrates that the closed-loop systems remain stable in the presence of a windgust as $\bar{q}$ and $M$ vary. Here, $\bar{q}$ varies linearly from 3.59 to $10.77 \mathrm{kPa}$ over $12 \mathrm{~s}$, and Mach varies from 0.5 to 0.82 as the function $(0.25+0.0352 t)^{\frac{1}{2}}$. This function was chosen taking into account the physical relationship between dynamic pressure and Mach. In other words air density was held nearly constant while still allowing Mach to vary only over the range for which linear models were available. The disturbance input is bandwidthlimited $(50 \mathrm{~Hz})$ white noise input to the gust model. Note that the variation in $\bar{q}$ and $M$ takes the plant through the region over which $K_{\mathrm{LFT}}$ was not designed (below $6.5 \mathrm{kPa}$ ). While the simulated system was in this region, $K_{\mathrm{LFT}}$ operated at $6.5 \mathrm{kPa}$ and at the simulated Mach. Thus the gain-scheduled controller remained on the edge of its operating range until $\bar{q}$ increased to $6.5 \mathrm{kPa}$.

Figure 7 demonstrates the greater disturbanceattenuationachieved by $K_{\mathrm{LFT}}$ in comparison to $K_{\mu}$, particularly for $t>4.8 \mathrm{~s}$ where the operating point has moved into the range for which $K_{\mathrm{LFT}}$ was designed. The peak leading- and trailing-edge accelerations for the simulation are 35.5 and $31.4 \mathrm{~cm} / \mathrm{s}^{2}$ for $K_{\mu}$ and 32.0 and $28.5 \mathrm{~cm} / \mathrm{s}^{2}$ for $K_{\mathrm{LFT}}$. For $t>4.8 \mathrm{~s}$ the rms accelerations of the leading- and trailing-edge flaps are 12.3 and $11.7 \mathrm{~cm} / \mathrm{s}^{2}$ for $K_{\mu}$ and 12.2 and $11.2 \mathrm{~cm} / \mathrm{s}^{2}$ for $K_{\mathrm{LFT}}$. Throughout $K_{\mathrm{LFT}}$ 's operating range it displays similar performanceimprovementin comparison to $K_{\mu}$. Simulations of both controllers at the LTI models in $K_{\mathrm{LFT}}$ 's operating range show an average of a $4 \%$ reduction in peak accelerationsand no significant improvement in rms accelerations. $K_{\mu}$, however, displays slightly
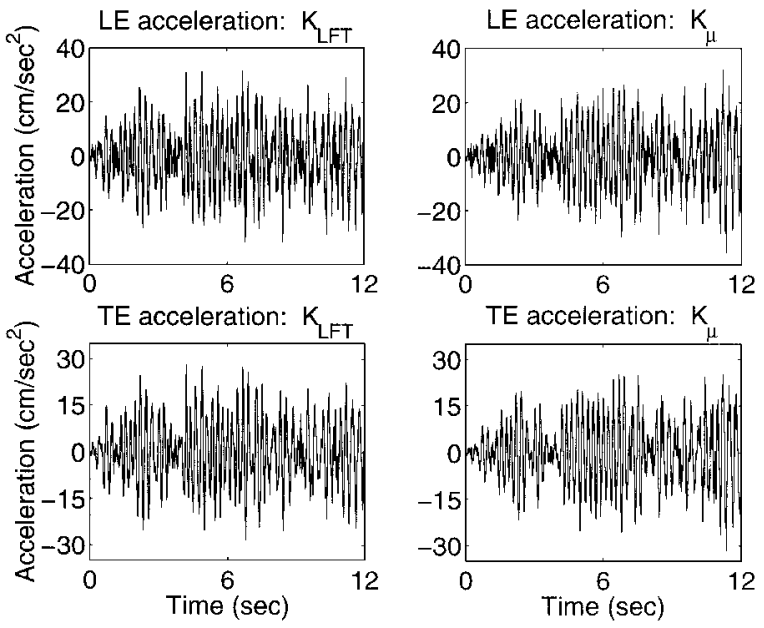

Fig. 7 Leading- and trailing-edge acceleration for $K_{\mathrm{LFT}}$ (left) and $K_{\mu}$ (right) as $\bar{q}$ and $M$ vary with time. 

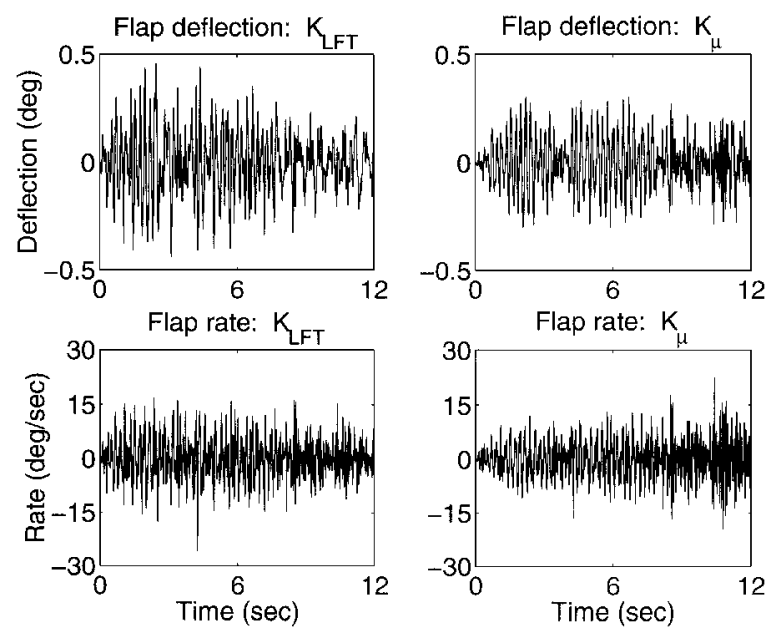

Fig. 8 Flap deflection and deflection rate for $K_{\mathrm{LFT}}$ (left) and $K_{\mu}$ (right) as $\bar{q}$ and $M$ vary with time.

better performance in terms of both peak and rms accelerations at points outside $K_{\mathrm{LFT}}$ 's operating range (below $6.5 \mathrm{kPa}$ ).

Figure 8 shows flap deflections and rates for $K_{\mathrm{LFT}}$ and $K_{\mu}$. Both controllersdemonstratereasonable actuator usage for the active flutter control problem. Flap deflections are limited to under $0.5 \mathrm{deg}$ whereas flap rates remain under $30 \mathrm{deg} \cdot \mathrm{s}^{-1} . K_{\mathrm{LFT}}$ appears to achieve better performance with smaller flap rates within its operating range. For $t>4.8 \mathrm{~s}$, the rms and peak flap rates for $K_{\mathrm{LFT}}$ are $5.17 \mathrm{deg} / \mathrm{s}$ and $16.16 \mathrm{deg} / \mathrm{s}$ whereas for $K_{\mu}$ they are $5.84 \mathrm{deg} / \mathrm{s}$ and $22.51 \mathrm{deg} / \mathrm{s}$, respectively. $K_{\mathrm{LFT}}$ control-surface deflections are slightly larger than deflections for $K_{\mu}$, but are still reasonable.

\section{Conclusions}

The LFT controller successfully meets both performance objectives. It stabilizes the wing over a large range of operating conditions and improves vibration attenuation. Both the maximum singular value plots and time simulations show the significant performance gains achievable through gain-scheduled control, in comparison not only to the open-loop system, but also to a linear $\mu$-synthesis controller.

The LFT control framework allows the control designer to take advantage of knowledge of how the physical system varies as a function of measurable parameters. Sending these parameters to the controllerin real time allows the controller to take advantage of this knowledge, resulting in improved performance.

Successful design relies upon intelligent choices of weighting functions and uncertainty models. The first-order multiplicative uncertainty limits the bandwidth of the controller, so that unmodeled high-frequencydynamics are not destabilizing. The LFT control design method is well suited to the flutter control problem, in which the plant dynamics change significantly but smoothly as a function of operating condition.

The gain-scheduled LFT controller was stable over the range of operation for which it was designed and by extension as a point controller, stabilized the model over the entire operating range. Both the gain-scheduled controller $K_{\mathrm{LFT}}$ and the linear controller $K_{\mu}$ increased the flutter boundary as measured in dynamic pressure by greater than $50 \%$ for Mach between 0.5 and 0.82 .

\section{Acknowledgments}

The authors wish to acknowledge the generous financial support from the U.S. Air Force Office of Scientific Research (AFOSR) under the Partnership for Research Excellence and Transition program (F49620-95-1-0419) and an AFOSR AASERT grant. The authors would also like to thank Carol Wieseman, NASA Langley Research Center, for letting us use the BACT data in our research.

\section{References}

${ }^{1}$ Theodorsen, T., "General Theory of Aerodynamic Instability and the Mechanism of Flutter,” NACA, Rept. 496, 1935.

${ }^{2}$ Kehoe, M. W., “A Historical Overview of Flight Flutter Testing," Pro- ceedings of Advanced Aeroservoelastic Testing and Data Analysis, AGARDCP-566, Rotterdam, The Netherlands, 1995, pp. 1.1-1.15.

${ }^{3}$ Waszak, M. R., and Srinathkumar, S., "Flutter Suppression for the Active Flexible Wing," Journal of Aircraft, Vol. 32, No. 1, 1995, pp. 61-67.

${ }^{4}$ Adams, W. M., Jr., and Christhilf, D. M., "Design and Multifunction Tests of a Frequency Domain-Based Active Flutter Suppression System," Journal of Aircraft, Vol. 32, No. 1, 1995, pp. 52-60.

${ }^{5}$ Bennet, R., Eckstrom, C., Rivera, J., Jr., Dansberry, B., Farmer, M., and Durham, M., "The Benchmark Aeroelastic Models Program-Description and Highlights of Initial Results,” NASA TM 104180, 1991.

${ }^{6}$ D'Cruz, J., "A Determination of the External Forces Required to Move the Benchmark Active Controls Testing Model in Pure Plunge and Pure Pitch," NASA TM 107743, 1993.

${ }^{7}$ Durham, M., Keller, D., Bennet, R., and Wieseman, C., "A Status Report on a Model for Benchmark Active Controls Testing," Proceedings of the 32nd AIAA/ASME/ASCE/AHS/ASC Structures, Structural Dynamics, and Materials Conference, AIAA, Washington, DC, 1991, pp. 704-709 (AIAA Paper 91-1011)

${ }^{8}$ Rivera, J., Jr., Dansberry, B., Bennet, R., Durham, M., and Silva, W., "NACA 0012 Benchmark Model Experimental Flutter Results with Unsteady Pressure Distributions," NASA TM 107581, 1992.

${ }^{9}$ Rivera, J., Jr., Dansberry, B., Farmer, M., Eckstrom, C., Seidel, D., and Bennet, R., "Experimental Flutter Boundaries with Unsteady Pressure Distributions for the NACA 0012 Benchmark Model," AIAA Paper 91-1010, April 1991.

${ }^{10}$ Scott, R., Hoadley, S., Wieseman, C., and Durham, M., "The Benchmark Active Controls Technology Model Aerodynamic Data," AIAA Paper 970829, Jan. 1997.

${ }^{11}$ Waszak, M., "Modeling the Benchmark Active Control Technology Wind-Tunnel Model Aerodynamic Data," AIAA Paper 96-3437, July 1996.

${ }^{12}$ Pototzky, A. S., Wieseman, C., Hoadley, S. T., and Mukhopadhyay, V., "On-Line Performance Evaluation of Multiloop Digital Control Systems," Journal of Guidance, Control, and Dynamics, Vol. 15, No. 4, 1992, pp. 878-883.

${ }^{13}$ Lichtenwalner, P. F., Little, G. R., Pado, L. E., and Scott, R. C., "Adaptive Neural Control for Active Flutter Suppression,' Proceedings of the ASME Fluids Engineering Division, American Society of Mechanical Engineers, New York, Vol. 242, 1996, pp. 3-8.

${ }^{14}$ Haley, P., and Soloway, D., "Generalized Predictive Control for Active Flutter Suppression," IEEE Control Systems, Vol. 17, No. 4, 1997, pp. 64-70.

${ }^{15}$ Lu, P. J., and Huang, L. J., "Optimal Control Law Synthesis for Flutter Suppression Using Active Acoustic Excitations," Journal of Guidance, Control, and Dynamics, Vol. 16, No. 1, 1993, pp. 124-131.

${ }^{16}$ Mason, G. S., and Berg, M. C., "Robustness of a Multirate Flutter Suppression System," Journal of Guidance, Control, and Dynamics, Vol. 16, No. 5, 1993, pp. 922-926.

${ }^{17}$ Ko, J., Kurdila, A. J., and Strganac, T. W., "Nonlinear Control Theory for a Class of Structural Nonlinearities in a Prototypical Wing Section," AIAA Paper 97-0580, Jan. 1997.

${ }^{18}$ Ozbay, H., and Bachmann, G. R., " $H_{2} / H_{\infty}$ Controller Design for a Two-Dimensional Thin Airfoil Flutter Suppression," Journal of Guidance, Control, and Dynamics, Vol. 17, No. 4, 1994, pp. 722-728.

${ }^{19}$ Tokur, O., and Ozbay, H., "Robustness Analysis of Controllers Designed for Active Flutter Suppression," Proceedings of the American Control Conference, Inst. of Electrical and Electronics Engineers, Piscataway, NJ, Vol. 1, 1995, pp. $182,183$.

${ }^{20}$ Fialho, I., Balas, G., Packard, A., Renfrow, J., and Mullaney, C., "Linear Fractional Transformation Control of the F-14 Aircraft Lateral-Directional Axis During Powered Approach Landing," Proceedings of the American Control Conference, Inst. of Electrical and Electronics Engineers, Piscataway, NJ, Vol. 1, 1997, pp. 128-132.

${ }^{21}$ Boyd, S., El Ghaoui, L., Feron, E., and Balakrishnan, V., Linear Matrix Inequalities in System and Control Theory. No. 15 in Studies in Applied Mathematics, Society for Industrial and Applied Mathematics, 1994.

${ }^{22}$ Gahinet, P., Arkadi, N., Laub, A. J., and Chilali, M., LMI Control Toolbox, MathWorks, Natick, MA, 1995, Chap. 8.

${ }^{23}$ Peele, E. L., and Adams, W. M., Jr., "A Digital Program for Calculating the Interaction Between Flexible Structures, Unsteady Aerodynamics, and Active Controls," NASA TM 80040, Jan. 1979.

${ }^{24}$ Adams, W. M., Jr., and Hoadley, S. T., "ISAC: A Tool for Aeroservoelastic Modeling and Analysis," NASA TM 109031, Dec. 1993.

${ }^{25}$ Blue, P., and Balas, G. J., "Linear Parameter-Varying Control for Active Flutter Suppression,” AIAA Paper 97-3640, Aug. 1997.

${ }^{26}$ Balas, G., Doyle, J., Glover, K., Packard, A., and Smith, R., $\mu$-Analysis and Synthesis Toolbox, MUS YN, Inc., Minneapolis, MN, 1995, Chaps. 4, 5.

${ }^{27}$ Zhou, K., Doyle, J., and Glover, K., Robust and Optimal Control, Prentice-Hall, Englewood Cliffs, NJ, 1996, pp. 271-300.

${ }^{28}$ Stein, G., and Doyle, J., "Beyond Singular Values and Loop Shapes," Journal of Guidance, Control, and Dynamics, Vol. 14, No. 1, 1991, pp. 5-16.

${ }^{29}$ Packard, A., "Gain Scheduling via Linear Fractional Transformations," Systems and Control Letters, Vol. 22, No. 2, 1994, pp. 79-92. 